\title{
Two-step kinetics of As/P exchange reaction
}

\author{
K. Y. Suh and Hong H. Lee ${ }^{a}$ \\ School of Chemical Engineering and Nanoelectronics Institute, Seoul National University, \\ Seoul 151-742, Korea \\ E. Yoon \\ School of Materials Science and Engineering, Seoul National University, Seoul 151-742, Korea
}

(Received 27 July 1998; accepted for publication 29 September 1998)

\begin{abstract}
A simple two-step mechanism is used to derive the kinetics of the As/P exchange reaction which takes place on an epitaxially grown InP surface exposed to As flux. The first step involves surface exchange of arsenic with phosphorus, which is then followed by the second step, bulk exchange of arsenic (arsenic incorporation). Two possible choices are investigated for bulk exchange: the same exchange rate constant in the bulk and the same ratio of exchange rate constants in the bulk. Transient and steady-state profiles of As composition and the maximum depth of the As/P exchange reaction are derived analytically. (C) 1999 American Institute of Physics. [S0021-8979(99)04601-0]
\end{abstract}

\section{INTRODUCTION}

The quality of heterointerfaces is of critical importance for high performance superlattice devices. Of these, material systems involving arsenic (As) and phosphorus (P) atoms such as $\mathrm{InGaAs} / \mathrm{InP}$ and $(\mathrm{Al}) \mathrm{GaInP} / \mathrm{GaAs}$ are technologically important for optoelectronic applications. ${ }^{1-9}$

The growth of these materials, however, is hampered by two major problems. One is the exchange of As with $\mathrm{P}, \mathrm{As} / \mathrm{P}$ exchange, ${ }^{1-7}$ the other is arsenic carryover. ${ }^{8,9}$ In typical growth experiments, the carryover is much less of a problem than the exchange since caution can be taken in the experiments to minimize the former problem whereas the latter is an inherent one.

The As/P exchange reaction occurs during heterostructural growth involving group $\mathrm{V}$ atoms. In the growth, the surface group $\mathrm{V}$ atoms are easily substituted by different group $\mathrm{V}$ atoms that impinge onto the surface and minimize the surface energy by forming the most stable chemical bond..$^{10}$ Experiments have shown that the As-containing surface layer stabilizes at around a two monolayer (ML) thickness. ${ }^{7}$ However, the thickness increases with increasing As exposure time, with the thickness being as deep as 5.5 ML for long exposure. ${ }^{3}$ But, according to scanning tunneling microscopy (STM) images, this may result from the roughness effects caused by many small islands on the surface. This surface exchange reaction deteriorates the metallurgical abruptness of the interface by creating a short range roughness that is smaller than the exciton diameter.

Although numerous studies have been carried out for As/P exchange, some of its key aspects including the exchange depth and the composition profile have not been studied theoretically. In this article, we propose a simple twostep kinetics mechanism for the As/P exchange that takes place on an epitaxially grown InP surface exposed to As flux.

a) Author to whom correspondence should be addressed; electronic mail: honghlee@plaza.snu.ac.kr

\section{TWO-STEP KINETICS}

The As/P exchange reaction is believed to occur in two steps. ${ }^{1-3}$ The first step involves adsorption of As followed by its incorporation into the surface layer by an exchange of As with P, which may also be called "surface exchange." The second is a bulk exchange step in which $\mathrm{P}$ in a given layer is exchanged with As in the adjoining two layers. According to this two-step mechanism, all the layers except for the surface layer are bulk.

According to the surface exchange concept, the rate of surface exchange should be equal to the rate of adsorption times the probability of exchange, i.e., $k_{d} C_{\mathrm{As}}\left(N_{s}-N_{1}\right)$ where $C_{\mathrm{As}}$ is the arsenic concentration in the gas phase, $N_{s}$ is the concentration of total surface sites, $N_{1}$ is the concentration of arsenic atoms in the first (surface) layer, and $k_{d}$ is the rate constant including the probability rate constant. As for bulk exchange involving the $n$th layer, the arsenic concentration in the layer increases as a result of the exchange from below the layer, $(n+1)$ th, and decreases as a result of the exchange to above the layer, $(n-1)$ th layer. Let $k_{e, n}$ be the rate constant for the probability of exchange from the $n$th layer to the $(n+1)$ th layer, which can be considered the forward exchange rate constant, and $k_{e, n}^{\prime}$ be the same for the exchange from the $n$th layer to the $(n-1)$ th layer, which can be called the backward exchange rate constant. Then, the exchange rate from the $n$th layer to the $(n+1)$ th layer, for example, can be written as $k_{e, n} N_{n}\left(N_{s}-N_{n+1}\right)$ since only $\left(N_{s}-N_{n+1}\right)$ sites are available in the $(n+1)$ th layer for the exchange.

The kinetics can then be written as follows:

$$
\begin{aligned}
\frac{d N_{1}}{d t}= & k_{d} C_{\mathrm{As}}\left(N_{s}-N_{1}\right)-k_{e, 1} N_{1}\left(N_{s}-N_{2}\right) \\
& +k_{e, 2}^{\prime} N_{2}\left(N_{s}-N_{1}\right) \quad(\text { for } n=1),
\end{aligned}
$$




$$
\begin{aligned}
\frac{d N_{n}}{d t}= & k_{e, n-1} N_{n-1}\left(N_{s}-N_{n}\right)-k_{e, n} N_{n}\left(N_{s}-N_{n+1}\right) \\
& -k_{e, n}^{\prime} N_{n}\left(N_{s}-N_{n-1}\right) \\
& +k_{e, n+1}^{\prime} N_{n+1}\left(N_{s}-N_{n}\right) \quad(\text { for } 2 \leqslant n<b), \\
\frac{d N_{b}}{d t}= & k_{e, b-1} N_{b-1}\left(N_{s}-N_{b}\right)-k_{e, b} N_{b}\left(N_{s}-N_{b+1}\right) \\
& -k_{e, b}^{\prime} N_{b}\left(N_{s}-N_{b-1}\right) \quad(\text { for } n=b),
\end{aligned}
$$

where the subscript $b$ is for the layer right above the bottommost layer in which there is no $\mathrm{P}$ exchange by As.

The kinetics equations can be solved for the profile as a function of time, given all the rate constants. However, much simpler and yet more effective relationships can be derived for two possible choices. The first is one in which all the exchange rate constants are the same except for the surface, i.e., the same value of exchange rate (SVER) constant for the bulk. For this choice, the rate equations reduce to

$$
\begin{aligned}
\frac{d f_{1}}{d t}= & k_{d} C_{\mathrm{As}}\left(1-f_{1}\right)-k_{e, 1} N_{s} f_{1}\left(1-f_{2}\right) \\
& +k_{e, 1} N_{s} f_{2}\left(1-f_{1}\right) \quad(\text { for } n=1) \\
\frac{d f_{n}}{d t}= & k_{e} N_{s}\left[f_{n-1}\left(1-f_{n}\right)-f_{n}\left(1-f_{n+1}\right)-f_{n}\left(1-f_{n-1}\right)\right. \\
& \left.+f_{n+1}\left(1-f_{n}\right)\right] \quad(\text { for } 2 \leqslant n<b) \\
\frac{d f_{b}}{d t}= & k_{e} N_{s}\left[f_{b-1}\left(1-f_{b}\right)-f_{b}\left(1-f_{b+1}\right)\right. \\
& \left.-f_{b}\left(1-f_{b-1}\right)\right] \quad(\text { for } n=b)
\end{aligned}
$$

where $k_{e}$ is now the exchange rate constant that is the same for $n \geqslant 2$ and $f_{n}\left(=N_{n} / N_{s}\right)$ is the fractional arsenic atom concentration in the $n$th layer. Note for $n=2$ that the backward rate constant $k_{e, 2}^{\prime}$ is different from $k_{e}$ but is the same as $k_{e, 1}$ since the exchange is toward the surface.

The steady-state arsenic profile and the maximum depth to which the exchange occurs follow directly from Eqs. (4)(6). The steady state profile is

$$
f_{n s}=f_{1 s}-\alpha\left(1-f_{1 s}\right)-(n-2) \beta\left(1-f_{1 s}\right) \quad(n \geqslant 2),
$$

where

$$
\begin{aligned}
& \alpha=k_{d} C_{\mathrm{As}} / N_{s} k_{e, 1}, \\
& \beta=k_{d} C_{\mathrm{As}} / N_{s} k_{e} .
\end{aligned}
$$

The fractional arsenic concentration in the surface layer, $f_{1 s}$, is an unknown and it must be specified for the calculation of $f_{n s}$. If the surface is exposed to As flux for an infinite amount of time, its value should be unity. However, the steady state profile obtained is usually for a finite amount of time and the value of $f_{1 s}$ is not unity but, rather, a value very close to unity. This value may be determined from experimental data. However, the value is set such that $1-f_{1 s}$ $=10^{-6}$ for the purpose of simulation and comparison throughout this article.
The maximum depth of As/P exchange can be obtained from Eq. (7) by setting $f_{n s}$ equal to zero

$$
n^{*}=n-1=\frac{f_{1 s}}{\beta\left(1-f_{1 s}\right)}-\gamma+1, \quad \gamma \equiv \alpha / \beta,
$$

where $n^{*}$ is the maximum depth. The parameter $\gamma$ is the ratio of exchange rate constant for the bulk to that for the surface. Its value is much less than unity since it is much more difficult for the bulk As/P exchange to take place compared with the surface exchange due to the lower mobility and higher strain barrier in the bulk. An example for which details are available is interdiffusion in a $\mathrm{Si} / \mathrm{Ge}$ heterostructure. It is known that the activation barrier is typically $3-4 \mathrm{eV}$ for $\mathrm{Ge}$ atoms in bulk $\mathrm{Si}$ or for $\mathrm{Si}$ in bulk Ge whereas the energy associated with surface segregation is $1-2 \mathrm{eV} .{ }^{11}$ If the same can be said about the InGaAs/InP system, ${ }^{12}$ the difference in activation energies is at least $1 \mathrm{eV}$ and the value of $\gamma$ at a typical growth temperature of $600{ }^{\circ} \mathrm{C}$ is of the order of $10^{-6}$.

The second choice is one in which the ratio of the forward exchange rate constant for the $n$th layer to that for the $(n+1)$ th layer, or $k_{e, n+1} / k_{e, n}$, is constant at $\gamma$, i.e., the same ratio of exchange rate (SRER) constants. Then the forward exchange rate constant for the $n$th layer is given by

$$
k_{e, n}=\gamma^{n-1} k_{e, 1} .
$$

From the definition, it follows that

$$
k_{e, n}^{\prime}=\frac{1}{\gamma} k_{e, n} .
$$

Therefore, Eqs. (1)-(3) for this choice reduce to

$$
\begin{aligned}
\frac{d f_{1}}{d t}= & k_{d} C_{\mathrm{As}}\left(1-f_{1}\right)-k_{e, 1} N_{s} f_{1}\left(1-f_{2}\right) \\
& +k_{e, 1} N_{s} f_{2}\left(1-f_{1}\right) \quad(\text { for } n=1), \\
\frac{d f_{n}}{d t}= & k_{e, 1} N_{s}\left\{\gamma^{n-2}\left[f_{n-1}\left(1-f_{n}\right)-f_{n}\left(1-f_{n-1}\right)\right]\right. \\
& \left.-\gamma^{n-1}\left[f_{n}\left(1-f_{n+1}\right)+f_{n+1}\left(1-f_{n}\right)\right]\right\} \\
& (\text { for } 2 \leqslant n<b), \\
\frac{d f_{b}}{d t}= & k_{e, 1} N_{s}\left\{\gamma^{b-2}\left[f_{b-1}\left(1-f_{b}\right)-f_{b}\left(1-f_{b-1}\right)\right]\right. \\
& \left.-\gamma^{b-1} f_{b}\left(1-f_{b+1}\right)\right\} \quad(\text { for } n=b) .
\end{aligned}
$$

The steady state profile follows from Eqs. (12)-(14) in the same manner as for the SVER:

$$
\begin{aligned}
& f_{n s}=f_{1 s}-\alpha\left(1-f_{1 s}\right)\left(k^{n-1}-1 / k-1\right), \\
& k \equiv 1 / \gamma .
\end{aligned}
$$

The maximum depth of As/P exchange is determined by Eq. (15) and it is given by

$$
n^{*}=n-1=\frac{\ln \left(1+\frac{(k-1) f_{1 s}}{\alpha\left(1-f_{1 s}\right)}\right)}{\ln k} .
$$

The transients of the fractional arsenic concentration in the $n$th layer, $f_{n}(t)$, are shown in Fig. 1 for both the SVER 


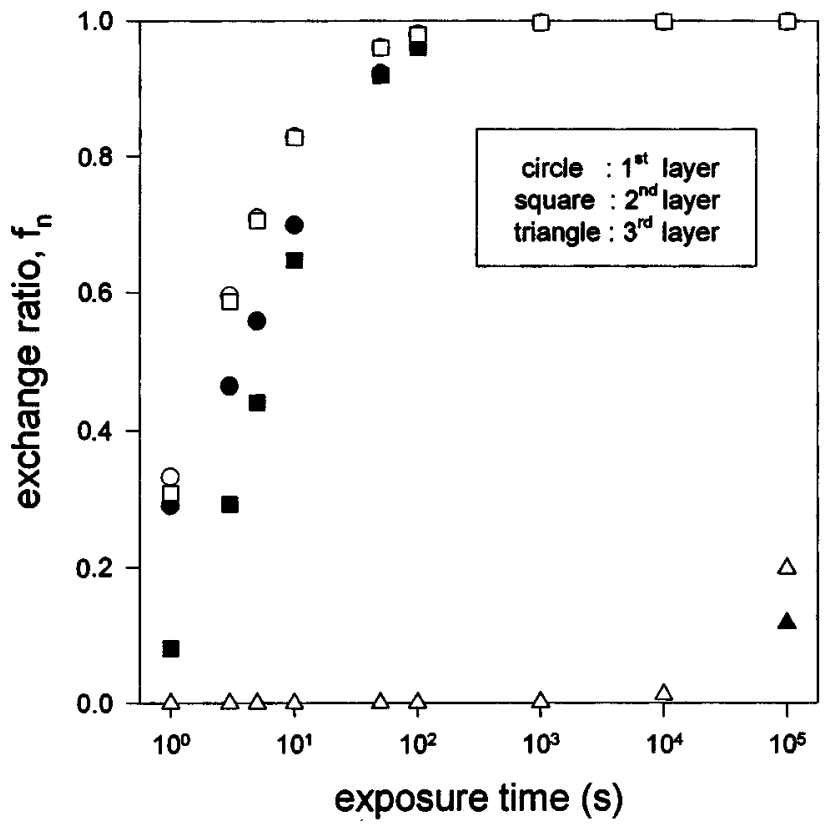

FIG. 1. Transient As profiles in underlying layers vs exposure time. The dark symbols are for the SRER and the open symbols are for the SVER. The parameter values are $\gamma=2.5 \times 10^{-6}$, and $k_{d} C_{\mathrm{As}}=k_{e, 1} N_{s}=0.5\left(\mathrm{~s}^{-1}\right)$.

and the SRER as a function of time. Although the time progression predicted by the SRER trails that by the SVER, the profiles in both cases rapidly reach the point of complete exchange $\left(f_{n}=1\right)$. However, it takes a long time for the exchange in the third layer to be noticeable. The steady state profiles are shown in Fig. 2. It is seen that As/P exchange occurs only up to $3 \mathrm{ML}$ in the case of the SRER, which is more realistic than the SVER, whereas it can reach up to 4.5 ML in the case of the SVER for an exchange rate constant

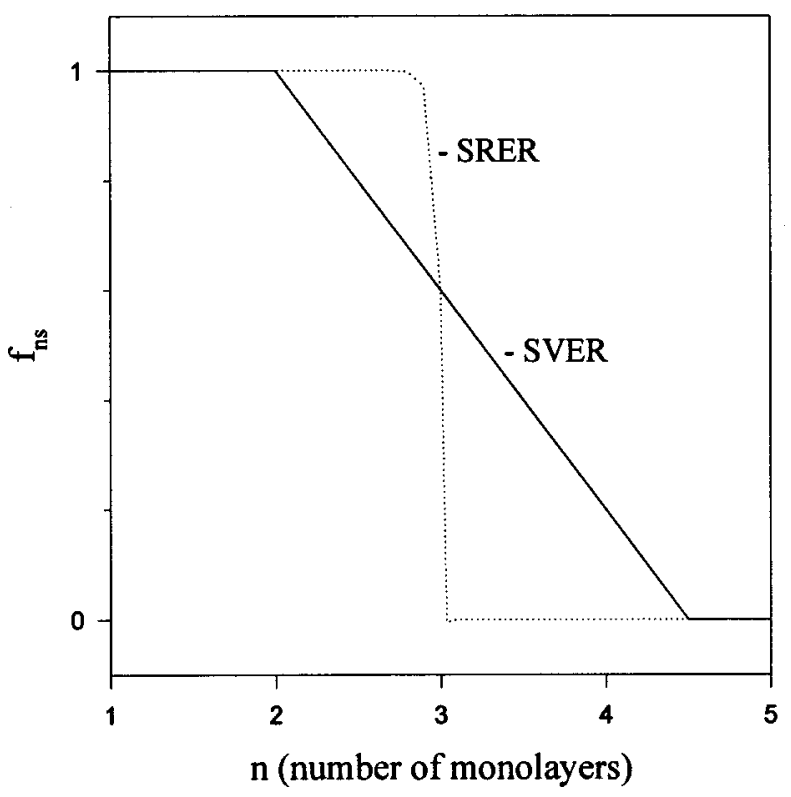

FIG. 2. Steady-state As profiles, $f_{n s}$, for $\alpha=1$ and $\beta=0.4 \times 10^{6}(\gamma=2.5$ $\left.\times 10^{-6}\right)$. Note that a continuous line is used instead of discrete data points for comparison purposes.

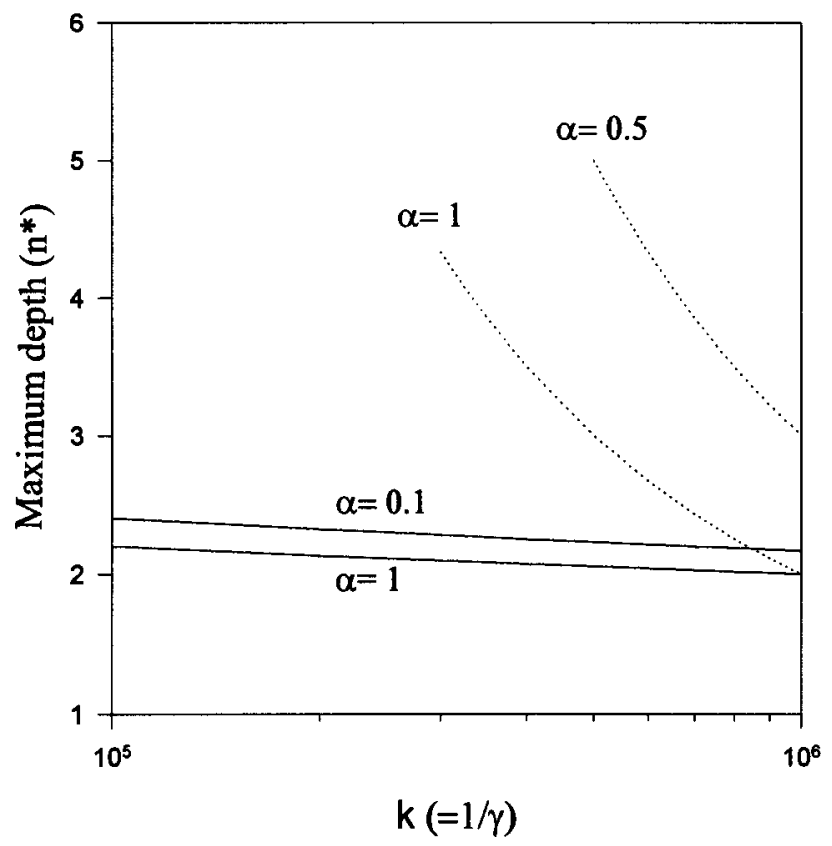

FIG. 3. Maximum depth of the As/P exchange reaction as a function of $\gamma$. The solid lines show the SRER and the broken lines the SVER.

ratio $(\gamma)$ of $2.5 \times 10^{-6}$. This maximum depth of As/P exchange is shown in Fig. 3 as a function of the inverse of exchange rate constant ratio. As the value of $\gamma$ approaches zero or as $k$ approaches infinity, the maximum depth of As/P exchange in terms of the number of ML is 2 and is typically 2-3 ML for practical ranges of parameters in the case of the SRER. However, in the case of the SVER, the maximum depth increases rapidly as $\gamma$ becomes larger, from which we can conclude that the SRER is more realistic for the As/P exchange reaction. Although now shown explicitly in Fig. 3, the steady-state penetration depth depends on temperature and $\mathrm{AsH}_{3}$ pressure, ${ }^{2,3}$ through parameters $\alpha$ and $k$. According to Eqs. (8) and (9), an increase in temperature results in a decrease in both $\alpha$ and $k$, which leads to an increase in the penetration depth as shown in Fig. 3. This trend is in agreement with that reported in the literature. ${ }^{2,3}$ The arsenic pressure, on the other hand, has the opposite effect on the depth. An increase in the pressure results in an increase in $\alpha$, which in turn has the effect of decreasing the penetration depth as revealed in Fig. 3, although the effect would be minimal. No definitive experimental results are available on the effects of arsenic pressure and therefore no comparison can be made at this time.

\section{COMPARISON WITH EXPERIMENTAL DATA}

Although the As/P exchange reaction has been studied extensively, ${ }^{1-2}$ experimental data in the literature for the transient As profile and the maximum exchange depth are rare. However, some comparisons can be made. Kobayashi and Kobayashi ${ }^{2}$ reported the $\mathrm{As} / \mathrm{P}$ exchange reaction monitored in situ by the surface photoabsorption (SPA) method on a submonolayer scale during metalorganic vapor phase epitaxial growth of a pseudomorphic InAs/InP heterostructure on (001) InP substrates. The dependence of the As/P 


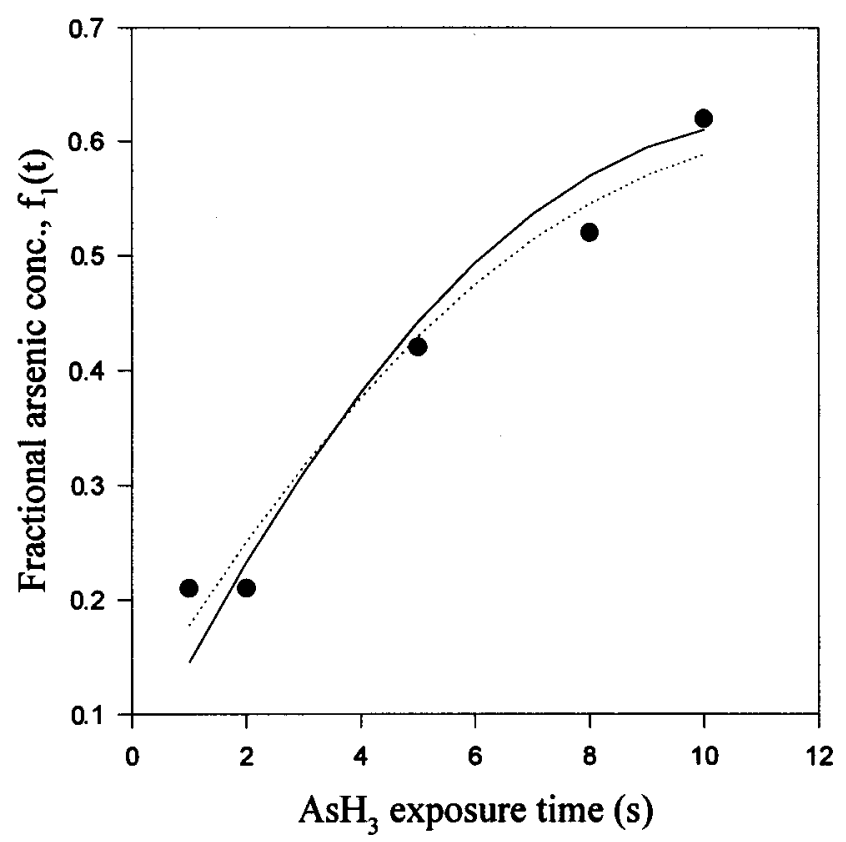

FIG. 4. Dependence of the $\mathrm{As} / \mathrm{P}$ exchange ratio on $\mathrm{AsH}_{3}$ exposure time along with our best-fit results using the SRER (solid line: $\gamma=10^{-6}$, $k_{d} C_{\mathrm{As}}=0.3 \mathrm{~s}^{-1}, k_{e, 1} N_{s}=0.6 \mathrm{~s}^{-1}$ ) and the SVER (broken line: $\gamma=10^{-6}$, $\left.k_{d} C_{\mathrm{As}}=0.16 \mathrm{~s}^{-1}, k_{e, 1} N_{s}=0.5 \mathrm{~s}^{-1}\right)$. The data are from Kobayashi and Kobayashi (Ref. 2).

exchange ratio on $\mathrm{AsH}_{3}$ exposure time is shown in Fig. 4 along with our best-fit results using the SVER and SRER. Although the SRER is more realistic than the SVER, it is seen that both models describe the fractional arsenic concentration almost equally well. It has been found that the exchange ratio is not sensitive to $N_{s} k_{e, 1}$ for either case (typically $0.5 \mathrm{~s}^{-1}$ ) but is sensitive to $k_{d} C_{\mathrm{As}}$.

\section{CONCLUSIONS}

The surface roughening of InP exposed to As flux has been investigated theoretically for the same value of exchange rate constant and the same ratio of exchange rate constants. The actual situation may exist between the SVER and the SRER, although the SRER is more realistic. Transient and steady-state profiles of As in the underlying layers and the maximum depth of the As/P exchange reaction have been derived analytically. Two-step kinetics of the As/P exchange reaction can be used to gain better understanding of conventional supperlattice growth involving different group $\mathrm{V}$ atoms.

${ }^{1}$ T. Anan, S. Sugou, and K. Nishi, Appl. Phys. Lett. 63, 1047 (1993).

${ }^{2}$ Y. Kobayashi and N. Kobayashi, Jpn. J. Appl. Phys., Part 1 31, 3988 (1992).

${ }^{3}$ B. X. Yang, L. He, and H. Hasegawa, J. Electron. Mater. 25, 379 (1995).

${ }^{4}$ Z. Sobiesierski, D. I. Westwood, P. J. Parbrook, K. B. Ozanyan, M. Hopkinson, and C. R. Whitehouse, Appl. Phys. Lett. 70, 1423 (1997).

${ }^{5}$ S. Mankefors, P. O. Nilsson, and J. Kanski, Phys. Rev. B 56, 15847 (1997)

${ }^{6}$ P. V. Santos, B. Koopmans, N. Esser, W. G. Schmidt, and F. Bechstedt, Phys. Rev. Lett. 77, 759 (1996).

${ }^{7}$ Z. Sobiesierski, D. I. Westwood, P. J. Parbrook, K. B. Ozanyan, M. Hopkins, and C. R. Whiteshouse, Appl. Phys. Lett. 70, 1423 (1997).

${ }^{8}$ W. Seifert, D. Hessman, X. Liu, and L. Samuelson, J. Appl. Phys. 75, 1501 (1994)

${ }^{9}$ T. Y. Wang, E. H. Reihlen, H. R. Jen, and G. B. Stringfellow, J. Appl. Phys. 66, 5376 (1989).

${ }^{10}$ J. M. Moison, M. Bensoussan, and F. Houzay, Phys. Rev. B 34, 2018 (1986).

${ }^{11}$ S. Fukatsu, K. Fujita, H. Yaguchi, Y. Shiraki, and R. Ito, Appl. Phys. Lett. 59, 2103 (1991)

${ }^{12}$ J. M. Sallese, S. Taylor, H. J. Bühlmann, J. F. Carlin, A. Rudra, R. Houdre, and Ilegems, Appl. Phys. Lett. . 65, 341 (1994). 